Check for updates

Cite this: RSC Adv., 2018, 8, 41012

Received 1st November 2018 Accepted 27th November 2018

DOI: $10.1039 / c 8 r a 09068 \mathrm{~h}$

rsc.li/rsc-advances

\section{Structural characterization of polysaccharides from Cordyceps militaris and their hypolipidemic effects in high fat diet fed mice $\dagger$}

\author{
Zhen-feng Huang, (D) \$ Ming-long Zhang, \$ Song Zhang, ${ }^{*}$ Ya-hui Wang \\ and Xue-wen Jiang
}

Cordyceps militaris is a crude dietary therapeutic mushroom with high nutritional and medicinal values. Mushroom-derived polysaccharides have been found to possess antihyperglycemic and antihyperlipidemic activities. This study aimed to partially clarify the structural characterization and comparatively evaluate hypolipidemic potentials of intracellular- (IPCM) and extracellular polysaccharides of $C$. militaris (EPCM) in high fat diet fed mice. Results indicated that IPCM-2 is $\alpha$-pyran polysaccharide with an average molecular weight of $32.5 \mathrm{kDa}$, was mainly composed of mannose, glucose and galactose with mass percentages of $51.94 \%, 10.54 \%$, and $37.25 \%$, respectively. EPCM-2 is an $\alpha$-pyran polysaccharide with an average molecular weight of $20 \mathrm{kDa}$ that is mainly composed of mannose, glucose and galactose with mass percentages of $44.51 \%, 18.33 \%$, and $35.38 \%$, respectively. In in vivo study, EPCM-1 treatment (100 $\mathrm{mg} \mathrm{kg}^{-1} \mathrm{~d}^{-1}$ ) showed potential effects on improving serum lipid profiles of hyperlipidemic mice, reflected by decreasing serum total cholesterol (TC), triglyceride (TG) and low density lipoprotein-cholesterol (LDL-C) levels by $20.05 \%, 45.45 \%$ and $52.63 \%$, respectively, while IPCM-1 treatment (100 mg kg $\mathrm{m}^{-1} \mathrm{~d}^{-1}$ ) remarkably decreased TC, TG and LDL-C levels by $20.74 \%, 47.93 \%$, and $38.25 \%$, respectively. In addition, EPCM-1 ameliorated hyperlipidemia possibly through upregulating the expression of serum lipoprotein lipase (LPL) and down-regulating the expression of hepatic 3-hydroxy3-methylglutaryl-CoA reductase (HMGR), as determined by enzyme-linked immunosorbent assay (ELISA) method, while IPCM-1 remarkably upregulated the expression of serum LPL. This study confirms polysaccharides from C. militaris could be explored as functional foods or natural medicines for preventing hyperlipidemia.

\section{Introduction}

Hyperlipidemia is the most common risk factor for developing fatty liver and cardiovascular disease, which are major health problems and account for approximately one third of all deaths worldwide. ${ }^{1}$ Clinically, elevated serum total cholesterol (TC), triglyceride (TG), low density lipoprotein-cholesterol (LDL-C), very low density lipoprotein-cholesterol (VLDL-C) levels, and decreased high density lipoprotein-cholesterol (HDL-C) level are risk factors for hyperlipidemia. ${ }^{2}$ Up until now, current chemical drugs used to treat hyperlipidemia, statins (e.g., lovastatin and simvastatin) and other hypolipidemic agents (e.g., acipimox and fibrates), are known to have modest clinical efficacy. However, they have adverse effects such as rhabdomyolysis and hepatotoxicity, that limit their application. ${ }^{3}$ Due to the limited and

School of Life Science, South China Normal University, No. 55 West of Zhongshan Avenue, Guangzhou, Guangdong, China.E-mail: zhangs@scnu.edu.cn

$\dagger$ Electronic supplementary information (ESI) available. See DOI: 10.1039/c8ra09068h

\$ These authors contributed equally. unsatisfactory therapeutic effects of antihyperlipidemic agents, using functional foods and nutraceuticals has been an alternative treatment to manage hyperlipidemia.

Most, if not all, mushrooms contain polysaccharides with various biological activities in their fruiting body, cultured mycelia and culture broth. Due to their multiple and effective biological functions as well as nontoxic characteristics, mushroom-derived polysaccharides, a kind of natural biological macromolecule known as "biological response modifiers", have received widespread clinical attention. ${ }^{\mathbf{4} 5}$ Cordyceps militaris, a valuable and rare edible-medicinal fungus belonging to the class Ascomycetes, has gained widespread popularity as a traditional medicine or nutraceutical due to its beneficial properties in healthy subjects, such as antitumor, immunomodulatory, antiinflammatory, antioxidative, hypouricemic, antinephritic, antihyperlipidemic, antibiotic and hepatoprotective effects. ${ }^{6-11}$ It contains multiple active components such as polysaccharides, cordycepin, adenosine, mannitol. ${ }^{11}$ As one of the major bioactive constituent of $C$. militaris, literature review shows that polysaccharides from $C$. militaris, exhibiting a potential for antioxidation, anti-inflammation and energy 
metabolism, are considered to possess beneficial effects on metabolic disorders such as hyperglycemia, hyperuricemic and hyperlipidemia, and therefore can function as novel potential hypolipidemic agents. ${ }^{11-16}$

Due to the advantages in more-compact culture space, shorter incubation time and higher production, currently, submerged fermentation has been used as a rapid and alternative method to accelerate the industrial level production of mushroom biomass and valuable metabolites. ${ }^{17-19}$ Recently, increasing evidences have indicated that the cordycepin and polysaccharides from fruiting bodies of cultured $C$. militaris show potential protection against hyperlipidemia, ${ }^{\mathbf{1 1}, 20}$ however, scarce literature about cultivated mycelia and fermented liquid has been published. Moreover, we found that extracellular polysaccharides released into culture medium during submerged fermentation of mushroom possessed a stronger biological properties than intracellular polysaccharides from mycelia in ours previous studies. ${ }^{21}$ To date, scarce investigation has been devoted to comparatively evaluate the hypolipidemic effects of polysaccharides from $C$. militaris mycelia and fermented liquid cultivated by submerged fermentation and underlying mechanisms. Furthermore, biological properties of polysaccharides were related with their molecular structure including monosaccharide composition, molecular weight, glycosidic bond, and conformation of the main chains. ${ }^{17,19}$ Gather up the threads, it is greatly significative and necessary to comparatively evaluate the effects of the intracellular- (IPCM) and the extracellular polysaccharides of $C$. militaris (EPCM) cultivated by submerged fermentation in preventing high-fat diet induced hyperlipidemia.

In this research, we focus on the preparation, structural characterization and hypolipidemic effects of polysaccharides from $C$. militaris. Firstly, two crude polysaccharides, IPCM-1 and EPCM-1, were isolated from the mycelia and fermented liquid of C. militaris, respectively. Afterward, two purified polysaccharides were obtained by column chromatography purification and characterized by Fourier transform-infrared spectroscopy (FT-IR), high performance liquid gel permeation chromatography (HPGPC), gas chromatography-mass (GC-MS) and circular dichroism (CD). In addition, the hypolipidemic activities of polysaccharides were evaluated using high-fat diet fed mice as model for exploration of clinical antihyperlipidemic mechanisms in pharmaceutical industry.

\section{Materials and methods}

\section{Materials}

Diethylaminoethyl (DEAE) -52 cellulose and Sephadex G-100 were obtained from Whatman. Co. (British). Dialysis tubes were obtained from Guangzhou Dongju Experimental Apparatus Co. Ltd. (Guangzhou, China). Trypsin 1:250 from porcine pancreas was purchased from Biosharp Biological Technology Co., Ltd (Hefei, China). Bovine serum albumin (BSA) and vitamin $\mathrm{B}_{1}$ were provided by Hangzhou Sijiqing Biological Engineering Materials Co. Ltd. (Hangzhou, China). All the other chemicals were of analytical grade.

\section{Fermentation process}

C. militaris used in the present study was donated by College of Life Science, South China Normal University (Guangzhou, Guangdong, China). The strain was maintained on slant medium (potato $200 \mathrm{~g}$, glucose $20 \mathrm{~g}$, peptone $1.0 \mathrm{~g},\left(\mathrm{NH}_{4}\right)_{2} \mathrm{SO}_{4}$ $1.0 \mathrm{~g}, \mathrm{KH}_{2} \mathrm{PO}_{4} 1.0 \mathrm{~g}, \mathrm{MgSO}_{4} \cdot 7 \mathrm{H}_{2} \mathrm{O} 1.0 \mathrm{~g}$ and agar $20 \mathrm{~g}$ per liter of distilled water, $\mathrm{pH} 6.5$ ), and incubated at $24{ }^{\circ} \mathrm{C}$ for 8 days. The $C$. militaris mycelia were transferred to $200 \mathrm{~mL}$ of sterile seed culture medium on a rotary shaker incubator at $26{ }^{\circ} \mathrm{C}$ and $160 \mathrm{rpm}$ to cultivate for 6 days. The seed medium and fermentation medium contained glucose $\left(50 \mathrm{~g} \mathrm{~L}^{-1}\right), \mathrm{KH}_{2} \mathrm{PO}_{4}$ $\left(2.0 \mathrm{~g} \mathrm{~L}^{-1}\right), \mathrm{NH}_{4} \mathrm{NO}_{3}\left(2.0 \mathrm{~g} \mathrm{~L}^{-1}\right), \mathrm{MgSO}_{4} \cdot 7 \mathrm{H}_{2} \mathrm{O}\left(1.0 \mathrm{~g} \mathrm{~L}^{-1}\right)$, and vitamin $\mathrm{B}_{1}\left(0.05 \mathrm{~g} \mathrm{~L}^{-1}\right)$. Seed culture obtained was cultivated at $27{ }^{\circ} \mathrm{C}$ in a $50 \mathrm{~L}$ fermentor (Baiou Equipment Co. Ltd, Guangzhou, China). The entire fermentor with $30 \mathrm{~L}$ fermentation medium was autoclaved at $121{ }^{\circ} \mathrm{C}$ for $30 \mathrm{~min}$. Fermentations were performed according the following conditions: initial $\mathrm{pH}, 6.5$; inoculum size, $7 \%$ (v/v); temperature, $27{ }^{\circ} \mathrm{C}$; aeration rate, $1 \mathrm{vvm}$; rotation speed, $150 \mathrm{rpm}$; tank pressure, 0.05-0.07 MPa; working time, $96 \mathrm{~h}$. The bioreactor culture obtained was transferred to a $500 \mathrm{~L}$ fermenter (Baiou Equipment Co. Ltd, Guangzhou, China) containing $300 \mathrm{~L}$ fermentation medium under the same conditions described above for another $96 \mathrm{~h}$.

\section{Extraction and characterization of IPCM-1 and EPCM-1}

Bioreactor culture obtained as described above was carried in ultra-speed centrifuge (GF-105 (B), Guangzhou GuangZhong Enterprise Group Corp, Guangzhou, China) to centrifuge at $15000 \mathrm{rpm}$ for $20 \mathrm{~min}$ to separately collect $C$. militaris mycelia and fermentation supernatant.

Fermentation supernatant was ultra-filtrated (polysulfone ultrafiltration membrane with a molecular cut off weight of 10 kDa, HeFei Shijie Membrane Engineering Co. Ltd, Hefei, China) to remove low-molecular-weight compounds (operating conditions: pressure, 10 bar; flux, 3-5 $\mathrm{L} \mathrm{min}^{-1}$; temperature, $22{ }^{\circ} \mathrm{C}$ ), followed by rotary evaporation under reduced pressure at $60{ }^{\circ} \mathrm{C}$ for further concentration. The broth culture filtrate was used to extract the EPCM-1. Mycelia were gathered, dried, crashed, sieved and defatted with $1: 2(\mathrm{w} / \mathrm{v})$ of petroleum ether. After drying at $50{ }^{\circ} \mathrm{C}$, mycelia were used to extract the IPCM- 1 .

The two polysaccharides were prepared according a previously reported method with some modification. ${ }^{22}$ The fermented liquid obtained were used to remove protein thoroughly by a combination of trypsin enzymolysis and Sevag method. ${ }^{23}$ Then the supernatant liquids were precipitated with 4 times volume of $95 \%(\mathrm{v} / \mathrm{v})$ ethanol, depigmented with $30 \%(\mathrm{v} / \mathrm{v}) \mathrm{H}_{2} \mathrm{O}_{2}$, and dialyzed against distilled water (6000-8000 Da molecular weight cut-off) for 3 days to obtain the EPCM-1. The mycelia were extracted three times with hot distilled water $(1: 25, \mathrm{w} / \mathrm{v})$ at $75{ }^{\circ} \mathrm{C}$ for $2.5 \mathrm{~h}$ followed by the purification processes described above for IPCM-1. A yield of $8.33 \%$ (lyophilized weight) watersoluble crude polysaccharides IPCM-1 were isolated from $C$. militaris mycelia, while crude polysaccharides EPCM-1 were prepared in a yield of $52.4 \mathrm{mg} \mathrm{L}^{-1}$ from fermented liquid. 


\section{Purification of IPCM-1 and EPCM-1}

Approximately $100 \mathrm{mg}$ of crude polysaccharides dissolved in $10 \mathrm{~mL}$ of Tris- $\mathrm{HCl}$ solution ( $\mathrm{pH}$ 8.0) were loaded on a DEAE-52 cellulose column $(3.0 \mathrm{~cm} \times 80 \mathrm{~cm})$, which were pre-equilibrated with Tris- $\mathrm{HCl}$ solution, and eluted in $0.4 \mathrm{M} \mathrm{NaCl}-$ Tris solution (pH 7.2) at a flow rate of $1 \mathrm{~mL} \min ^{-1}$ (6 mL per tube). The products obtained were further purified by size-exclusion chromatography on a Sephadex G-100 column $(3.0 \mathrm{~cm} \times 80$ $\mathrm{cm})$ and eluted with $1 \mathrm{M} \mathrm{NaCl}$-Tris solution ( $\mathrm{pH} \mathrm{8.0)}$ at a flow rate of $1 \mathrm{~mL} \mathrm{~min}^{-1}$ ( $6 \mathrm{~mL}$ per tube). Each elute was monitored for carbohydrate determination via using the phenol-sulfuric acid method with D-glucose as a standard. ${ }^{24}$ The same carbohydrate-positive fractions were pooled together, dialyzed, and lyophilized. The lyophilized samples of two purified polysaccharides, termed IPCM-2 and EPCM-2, were white powders and they showed positive responses to Molisch test, and negative reactions to iodine-potassium iodide, Fehling's reagent and ferric trichloride, ninhydrin and Bradford test, which indicates the absence of starch-type polysaccharides, reducing sugars and phenolics, amino acids and proteins in IPCM-2 and EPCM-2..23,25 The percentage of total carbohydrate content was $91.63 \%$ in IPCM-2 and 91.68\% in EPCM-2.

\section{UV and FT-IR spectrometric analysis}

The polysaccharide solution $\left(1 \mathrm{mg} \mathrm{mL}^{-1}\right)$ was prepared and applied to ultraviolet spectral analysis. The UV scanning spectrum was measured in the wavelength range of $190-600 \mathrm{~nm}$ by spectrophotometer (UV-2400, Shimadzu, Japan).

The absorption spectrum of the IPCM-2 and EPCM-2 were obtained using FT-IR spectroscopy. $10 \mathrm{mg}$ of the sample was thoroughly ground with $\mathrm{KBr}$ powder (100 mg). The dried mixture was then pressed into $1 \mathrm{~mm}$ thick pellets for FT-IR spectral analysis. The FT-IR spectra were recorded in a range of $4000-450 \mathrm{~cm}^{-1}$ by an transmission infrared spectrometer (Sigma, USA).

\section{Molecular weight distribution analysis}

The molecular weight and homogeneity of IPCM-2 and EPCM-2 were measured by HPGPC, which was operated with a HPLC system (1100, Agilent Technologies, USA) equipped with a refractive index detector (RID, detecting temperate $35^{\circ} \mathrm{C}$ ) and a TSK-GEL G3000SW XL column $(300 \mathrm{~mm} \times 718 \mathrm{~mm}) .{ }^{19}$ $\mathrm{NaH}_{2} \mathrm{PO}_{4}-\mathrm{Na}_{2} \mathrm{HPO}_{4}$ aqueous solution (0.05 M, pH 6.7) was used as mobile phase at a flow rate of $0.8 \mathrm{~mL} \mathrm{~min}{ }^{-1}$. Standard dextrans with different molecular weights ( $M$ 5.8, 12.2, 23.7, 48, $100,186,380,853 \mathrm{kDa}$ ) were used for comparison to calculate the standard curve. The relationship between $\log M$ and retention time $T$ was calculated as the following equation:

$$
\log M=-0.2524 T+7.5766 R^{2}=0.982
$$

\section{Monosaccharide composition analysis}

GC-MS spectrometry was applied for the identification and quantification of monosaccharides of IPCM-2 and EPCM-2. ${ }^{26}$ Briefly, $2 \mathrm{M}$ sulfuric acid was used to hydrolyze polysaccharide at $100{ }^{\circ} \mathrm{C}$ for $6 \mathrm{~h}$. Barium hydroxide was used to adjust $\mathrm{pH}$ of hydrolyzed polysaccharide to neutrality and then the mixture was evaporated at $45^{\circ} \mathrm{C}$. The hydrolysate was dissolved in $5 \mathrm{~mL}$ of pyridine with $70 \mathrm{mg}$ of hydroxylamine hydrochloride in a tube and reacted at $90^{\circ} \mathrm{C}$ for $1 \mathrm{~h}$. After cooling, $5 \mathrm{~mL}$ of acetic anhydride was added and the mixture was incubated at $90^{\circ} \mathrm{C}$ for $1 \mathrm{~h}$, then analyzed by GC-MS on a Agilent 6890GC/5973MS chromatograph equipped with a fused silica capillary column $(15 \mathrm{~mm} \times 0.2 \mathrm{~mm})$. The standard chromatograms of ribose, rhamnose, arabinose, xylose, mannose, glucose and galactose were used for composition identification of IPCM-2 and EPCM-2 (chemical structures of monosaccharides see Fig. S1†), and the relative mass percentages were calculated by the area normalization method.

\section{CD spectrum analysis}

CD spectrum was employed to analyze the conformation IPCM2 and EPCM-2. ${ }^{27}$ The polysaccharide samples dissolved in deionized water $\left(0.05 \mathrm{mg} \mathrm{mL}^{-1}\right)$ was kept at $25{ }^{\circ} \mathrm{C}$ for $2 \mathrm{~h}$. The mixtures were analyzed by CD spectrometer (J-500, Jasco Corporation, Japan). Data was collected in a range of 190$350 \mathrm{~nm}$ at $1 \mathrm{~nm}$ interval.

\section{Congo red analysis}

The conformational structures of the polysaccharides were determined by Congo red analysis referenced reported method. ${ }^{28}$ Firstly, polysaccharide solution $\left(2.5 \mathrm{mg} \mathrm{mL}^{-1}\right)$ was mixed with the same volume of Congo red aqueous solution (80 $\mu \mathrm{M})$. Then, $\mathrm{NaOH}$ solution ( $1 \mathrm{M}$ ) were added to bring a final $\mathrm{NaOH}$ concentrations to $0,0.10,0.20,0.30,0.40$ and $0.50 \mathrm{M}$. After incubating at $25{ }^{\circ} \mathrm{C}$ for $10 \mathrm{~min}$, these mixtures were employed to ultraviolet spectral analysis in the wavelength range of $400-800 \mathrm{~nm}$ find the $\lambda_{\max }$ (maximum absorption wavelength). The process above was carried out using pure water instead of polysaccharide solution as a control.

\section{Animals, diets, and experimental procedures}

Allowing for the low efficiency and yield of column chromatographic purification, and the large amount of IPCM-2 and EPCM-2 in IPCM-1 and EPCM-1, respectively, a large dosage of IPCM-1 and EPCM-1 were used to investigate hypolipidemic activities of IPCM-2 and EPCM-2 in mice fed a high-fat diet. ${ }^{29}$ Ninety adult male Kunming (KM) mice, weighting $20 \pm 2 \mathrm{~g}$, were obtained from the Experimental Animal Center of Sun Yat-sen University (Guangzhou, Guangdong, China) [Certificate No. SCXK (Yue) 2011-0029]. During the experiment, the mice were housed in individual stainless-steel cages and maintained at 20 $\pm 2{ }^{\circ} \mathrm{C}$ under a light/dark cycle of $12 \mathrm{~h}$ and relative humidity 55 $\pm 5 \%$. All mice were acclimated to laboratory conditions for 4 days before the onset of the experiment and they had free access to rodent laboratory food and drinking water. Simvastatin tablet 
from Hangzhou Merck \& Co., Inc. (Approval number: J20090001) was used as a positive control drug. ${ }^{17,19}$ The mice as mentioned above were randomly divided into 9 groups, with 10 mice in each group received the following treatment schedule (Fig. 1):

Normal control (NC), mice fed a standard diet with daily oral gavage $10 \mathrm{~mL} \mathrm{~kg}{ }^{-1}$ body weight (BW) distilled water.

Hyperlipidemic control (HC), mice fed a high-fat diet with oral gavage $10 \mathrm{~mL} \mathrm{~kg}{ }^{-1}$ BW distilled water daily.

Positive control (PC), fed with high-fat diet and received in addition $10 \mathrm{mg} \mathrm{kg}^{-1} \mathrm{BW}$ of simvastatin daily.

L-IPCM-1, M-IPCM-1 and H-IPCM-1: mice fed a high-fat diet and supplemented with IPCM-1 through daily gavage at doses of 50,100 , and $200 \mathrm{mg} \mathrm{kg}^{-1} \mathrm{BW}$, respectively.

L-EPCM-1, M-EPCM-1 and H-EPCM-1: mice fed a high-fat diet and supplemented with EPCM-1 through daily gavage at doses of 50,100 , and $200 \mathrm{mg} \mathrm{kg}^{-1}$ BW, respectively.

The feed for the experimental animals was supplied by Guangdong Medical Laboratory Animal Center (Guangzhou, Guangdong, China) [Certificate No. SCXK (Yue) 2008-0002]. The high-fat diet consisted of $1.0 \%$ cholesterol (w/w), $10 \%$ lard, $0.3 \%$ bile salt, and $88.7 \%$ standard diet. The animal experiment was lasted for 28 consecutive days. Body weight was measured weekly and food intake adjusted until the end of the experiment. At the end of experiment, all mice were fasted overnight and sacrificed by clavicle dislocation after anesthetizing with sodium thiopental. Blood samples were collected in heparinized tubes and immediately separated for acquisition of serum by centrifugation at $4{ }^{\circ} \mathrm{C}$ and $3000 \mathrm{rpm}$ for $15 \mathrm{~min}$, and then stored at $-80{ }^{\circ} \mathrm{C}$ until biochemical analysis. After blood collection, the heart, liver, kidney and spleen were excised immediately, wiped by filter paper and weighed after being rinsed with ice-cold phosphate buffered saline (PBS). Organ indexes were defined as the weight of organs divided by the weight of mice. Liver tissue was homogenized with ice-cold PBS and ten percent $(\mathrm{w} / \mathrm{v})$ of liver homogenate was centrifuged at $4{ }^{\circ} \mathrm{C}$ and $4000 \mathrm{rpm}$ for $10 \mathrm{~min}$. The liver supernatant obtained after centrifugation twice was stored at $-20^{\circ} \mathrm{C}$ until assay.

All experimental procedures were performed in accordance with the Guidelines for Animal Care established by the National Institute of Health, and approved by the Ethics Committee for animal research at South China Normal University.

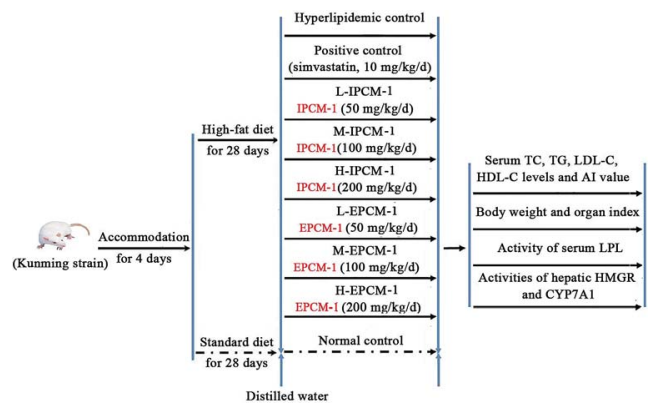

Fig. 1 Animal experimental procedure.

\section{Determination of serum lipid profiles}

Serum lipids level indices of TC, TG, LDL-C, and HDL-C were measured using commercial assay kits purchased from Nanjing JianCheng Institute of Bioengineering (Nanjing, China). TC and TG levels were quantified with colorimetric enzymatic method by the commercial kit CHOD-PAP-enzymatic-colorimetric and GPO-PAP-enzymatic-colorimetric, respectively. ${ }^{30}$ HDL-C and LDL-C levels were measured using direct enzymatic commercial assay kits. ${ }^{31}$ The results were expressed in $\mathrm{mmol} \mathrm{L}^{-1}$ of serum. The atherogenic index (AI) was calculated as the following formula: ${ }^{32}$

$$
\mathrm{AI}=\frac{\mathrm{TC}}{\mathrm{HDL}-\mathrm{C}}
$$

\section{Determination of serum LPL activity}

LPL can catalyze the decomposition of core TG in chylomicrons and VLDL-C into free fatty acids. ${ }^{33}$ The activity of serum LPL was measured using commercial assay kits purchased from the Nanjing Jiancheng Institute of Bioengineering (Nanjing, Jiangsu, China) according to the manufacturer's instructions, which was quantified based on the free fatty acid production. Briefly, the sample was reacted with substrate solution of assay kits at $37{ }^{\circ} \mathrm{C}$ for $20 \mathrm{~min}$, then the content of free fatty acids of samples was detected with copper reagent method by iMARK Microplate Reader (Bio-Rad Laboratories, Hercules, CA, USA), which have a maximum absorbance at $550 \mathrm{~nm}$ in UV-vis spectrophotometer. The controls were treated with the same quantity of double distilled water. The activity of serum LPL $\left(A_{\mathrm{LPL}}\right)$ was calculated using the following equation:

$$
A_{\mathrm{LPL}}=\frac{\mathrm{OD}_{\text {serum }}-\mathrm{OD}_{\text {control }}}{\mathrm{OD}_{\text {standard }}-\mathrm{OD}_{\text {control }}} \times C_{\text {standard }} \times V
$$

where $\mathrm{OD}_{\text {serum }}, \mathrm{OD}_{\text {control }}$ and $\mathrm{OD}_{\text {standard }}$ are the optical density (OD) value at $550 \mathrm{~nm}$ of the serum sample, the control and LPL standard, respectively, $C_{\text {standard }}$ is the concentration of LPL

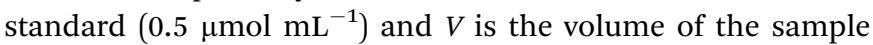
(mL). $A_{\mathrm{LPL}}$ was recorded in $\mathrm{U} \mathrm{mL}^{-1}$ of serum. One unit of LPL activity was defined as $1 \mu \mathrm{mol}$ of free fatty acids released in $1 \mathrm{~mL}$ of serum with a $1 \mathrm{~h}$ reaction time.

\section{Determination of hepatic HMGR and CYP7A1 activities by sandwich enzyme-linked immunosorbent assay (ELISA)}

Hepatic HMGR and CYP7A1 activities were measured using standard sandwich ELISA kits (R\&D, USA) according to the manufacturer's instructions. Briefly, assay plates were coated with mouse anti-HMGR or anti-CYP7A1 antibody and blocked with $5 \%$ skim milk at $25{ }^{\circ} \mathrm{C}$ for $2 \mathrm{~h}$. Then the plates were reacted with $50 \mu \mathrm{L}$ liver supernatant samples diluted with diluent solution $(1: 4 \mathrm{v} / \mathrm{v})$ at $37^{\circ} \mathrm{C}$. After reactions for $30 \mathrm{~min}$, the plates were washed five times using the wash buffer. The captured HMGR or CYP7A1 was detected using biotin-antibody, together with horseradish peroxidase (HRP)-conjugated avidin. After washing five times with the wash buffer, color reactions were reacted with $3,3^{\prime}, 5,5^{\prime}$-tetramethylbenzidine (TMB) substrate and 
measured at $450 \mathrm{~nm}$. Two enzyme standards with different concentration were used to calibrate the standard curve. HMGR and CYP7A1 activities of samples were determined according to the standard curves and the results were recorded in $\mathrm{U} \mathrm{L}^{-1}$ of liver supernatant.

\section{Statistical analysis}

The data were expressed as means \pm standard deviation (SD) and determinations were obtained from ten animals per group. The one-way analysis of variance (ANOVA) followed by Student's $t$-test was used to detect significant differences between any two groups. $P$-value $<0.05$ were regarded as statistically significance.

\section{Results and discussion}

\section{Spectrometric analysis}

The UV spectrum of IPCM-2 and EPCM-2 were presented in Fig. 2A and B. No absorption was occurred at 260 and $280 \mathrm{~nm}$ in the ultraviolet spectrum of IPCM-2 and EPCM-2, indicating absence of nucleic acids and protein contained. ${ }^{19}$ The results were exactly consistent with the determination in protein content by Bradford assay and further showed that DEAE-52 cellulose column selected were effective.

The FT-IR spectroscopy of IPCM-2 and EPCM-2 were showed in Fig. 2C and D. We can find that IPCM-2 and EPCM-2 showed similar absorption bands in the $450-4000 \mathrm{~cm}^{-1}$ region which are admitted as the characteristic peaks of polysaccharides. ${ }^{17,26}$ Specifically, the broad stretching peaks at 3346 (IPCM-2) and 3370 (EPCM-2) $\mathrm{cm}^{-1}$ were ascribed to the hydroxyl groups with stretching vibration. The absorption bands at about $2930 \mathrm{~cm}^{-1}$ (IPCM-2) and $2933 \mathrm{~cm}^{-1}$ (EPCM-2) were attributed to $\mathrm{C}-\mathrm{H}$ stretching vibrations of the polysaccharides. The strong band at $1651 \mathrm{~cm}^{-1}$ was derived from $\mathrm{C}=\mathrm{O}$ vibration, and the weak bond at about $1415 \mathrm{~cm}^{-1}$ was originated from $\mathrm{C}-\mathrm{H}$ bending vibration. The two bonds at $1034 \mathrm{~cm}^{-1}$ (IPCM-2) and $1039 \mathrm{~cm}^{-1}$ (EPCM-2) were the characteristic of $\mathrm{C}-\mathrm{O}-\mathrm{C}$, suggesting the presence of pyranose sugars. Moreover, characterization of IPCM-2 and IPCM-2 showed the typical absorption of D-pyran ring grape
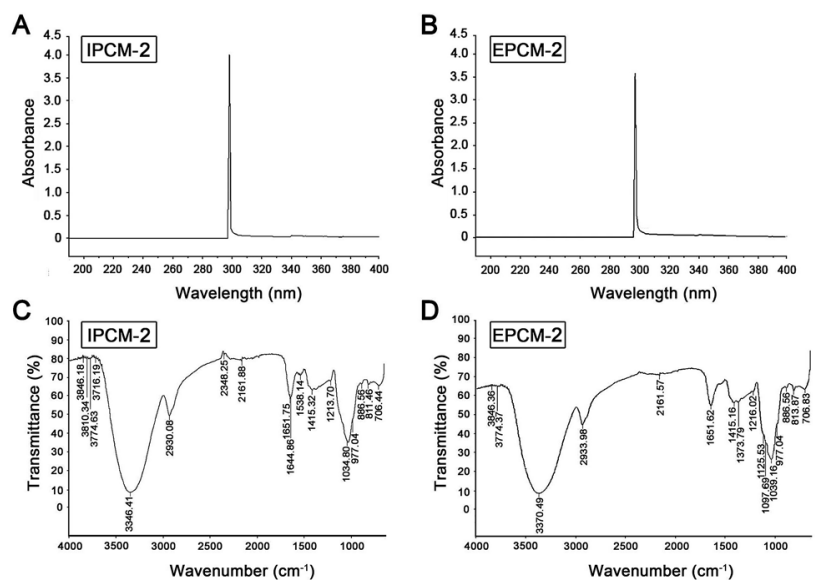

Fig. 2 Spectrometric analysis. UV spectrum of IPCM-2 (A) and EPCM2 (B); FT-IR spectrum of IPCM-2 (C) and EPCM-2 (D). asymmetric stretching vibration at round $977 \mathrm{~cm}^{-1}$ and symmetric stretching vibration at round $706 \mathrm{~cm}^{-1}$. The absorption bands centered at 811 (IPCM-2) and 813 (EPCM2) $\mathrm{cm}^{-1}$ were due to the $\alpha$-type glycosidic linkages. Thus, IPCM2 and EPCM- 2 are both $\alpha$-pyran polysaccharides.

\section{Molecular weight and monosaccharide composition}

As shown in Fig. 3, a single symmetrical peak was found in the HPGPC profiles of IPCM-2 and EPCM-2, indicating that they are both homogeneous polysaccharides. The weight-average molecular weight $\left(M_{\mathrm{w}}\right)$ and number-average molecular weight $\left(M_{\mathrm{n}}\right)$ of IPCM-2 was 32.5 and $16.1 \mathrm{kDa}$, respectively. The $M_{\mathrm{w}}$ and $M_{\mathrm{n}}$ of EPCM-2 was 20 and $13.4 \mathrm{kDa}$, respectively. The $M_{\mathrm{w}} / M_{\mathrm{n}}$ values of IPCM-2 and EPCM-2 were 2.02 and 1.49, respectively, suggesting that IPCM-2 and EPCM-2 are polysaccharide polymers.

The monosaccharide compositions of IPCM-2 and EPCM-2 were analyzed by means of comparing the retention time with monosaccharide guide samples (Fig. 4). The IPCM-2 was composed of seven different monosaccharides including ribose, rhamnose, arabinose, xylose, mannose, glucose and galactose with a mass percentage of $0.012 \%, 0.066 \%, 0.11 \%, 0.08 \%$, $51.94 \%, 10.54 \%$ and $37.25 \%$, respectively, while the EPCM-2 was composed of ribose, rhamnose, arabinose, xylose, mannose, glucose and galactose in a percentage composition of $0.14 \%, 0.35 \%, 0.57 \%, 0.72 \%, 44.51 \%, 18.33 \%$ and $35.38 \%$, respectively. Obviously, the main difference between the two polysaccharides is the amount of glucose, whose content in EPCM-2 is markedly higher than that in IPCM-2. The monosaccharide compositions analysis revealed that the major components of IPCM-2 and EPCM-2 were mannose, glucose and galactose, and minor contents of ribose, rhamnose, arabinose and xylose were also noted, which was consistent with previous studies. ${ }^{4,5}$

\section{Spatial conformation studies}

From the results of the CD spectra (Fig. 5A and B), CD signals of IPCM-2 and EPCM-2 appeared at $200 \mathrm{~nm}$, showed negative cotton effect, indicating that IPCM-2 and EPCM-2 were asymmetric molecules. Congo red can form complexes with a helical

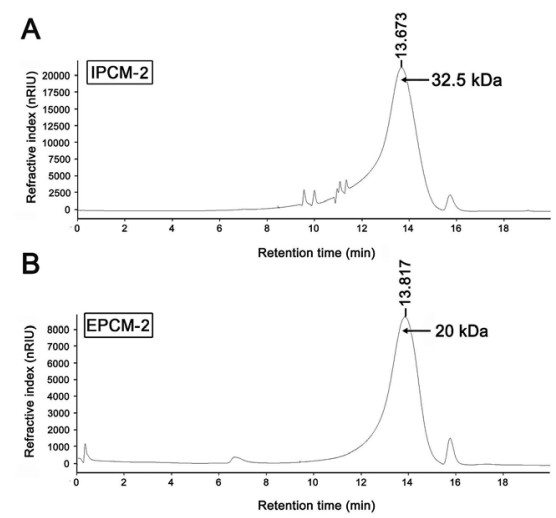

Fig. 3 High performance liquid gel permeation chromatography chromatogram of IPCM-2 (A) and EPCM-2 (B). 
A
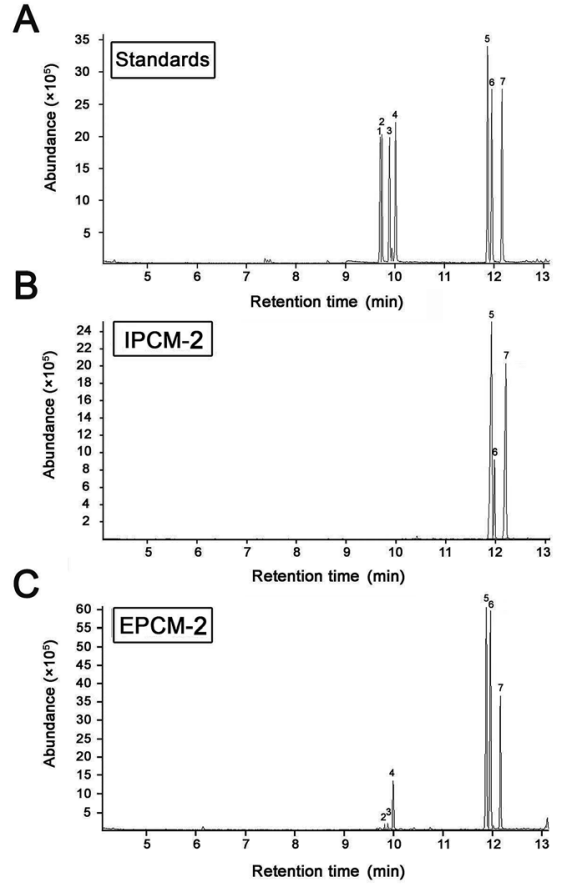

Fig. 4 Gas chromatography-mass chromatograms of monosaccharide standard samples (A), IPCM-2 (B) and EPCM-2 (C). Peaks: (1) ribose, (2) rhamnose, (3) arabinose, (4) xylose, (5) mannose, (6) glucose and (7) galactose.

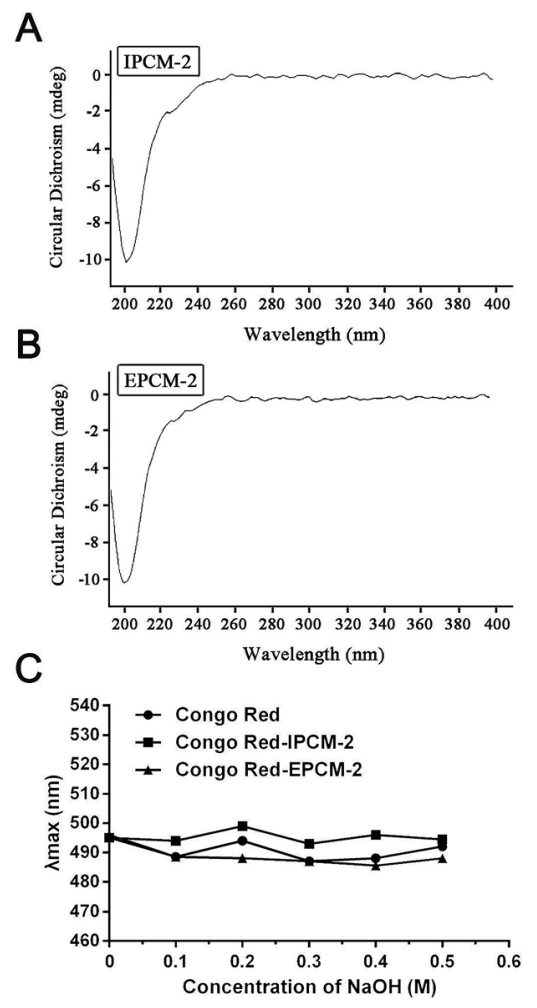

Fig. 5 Conformation studies of IPCM-2 and EPCM-2. (A): Circular dichroism spectra of IPCM-2; (B): circular dichroism spectra of EPCM2 ; $(C)$ : result of the maximum absorption wavelength $\left(\lambda_{\max }\right)$ of Congo red test. conformation of polysaccharides when Congo red is mixed under alkaline conditions, and $\lambda_{\max }$ of the complex will show bathochromic shift in the wavelength range of $400-600 \mathrm{~nm}$ as compared with pure Congo red. ${ }^{34}$ However, the $\lambda_{\max }$ of IPCM-2and EPCM-2-Congo red complexes had no bathochromic shift compared with control group, indicating that IPCM-2 and EPCM-2 did not exhibit a triple-helical conformation at alkali concentrations of 0 to $0.5 \mathrm{M}$ and showed random coil conformations (Fig. 5C). These results were in accordance with Lee's reports. ${ }^{4,5}$

\section{Effects of IPCM-1 and EPCM-1 on serum lipid profiles}

High TC and TG are risk factors that can cause hyperlipidemia and several literatures have reported that alleviating the elevated serum TC and TG levels is quite beneficial for the amelioration of hyperlipidemia. ${ }^{17}$ In addition, elevated serum TG and LDL-C levels are proposed to be related with increased risk of coronary heart disease. ${ }^{35}$ Effects of IPCM-1 and EPCM-1 on serum lipid profiles of hyperlipidemic mice were examined and results are shown in Fig. 6. It is clear that compared with NC mice, the serum levels of TC, HDL-C and LDL-C in HC mice were significantly higher, whereas the serum TG level was remarkably lower presumably because dietary administration of high-fat diet cause non-alcoholic fatty liver disease (NAFLD), which involves increased TG uptake and decreased lipid export in the liver. ${ }^{36}$ It indicated that high-fat diet feeding resulted in obvious lipid metabolism disorder in the KM mice and made them hyperlipidemia mice. In vivo study, positive simvastatin, a high efficiency antihyperlipidemic drug, was manifested potential effects against the increase of serum TC $(11.98 \%$ decrease, $p<0.05)$, TG $(25.62 \%$ decrease, $p<0.01)$ and LDL-C $(16.14 \%$ decrease, $p<0.01)$ levels induced by high-fat diet, whereas no obvious effect in HDL-C level. Interestingly, the serum levels of TC, TG and LDL-C in the IPCM-1 and EPCM-1 treated group were remarkably lower than in the PC group. As presented in Fig. 6, in the dosage groups treated with IPCM-1 at 50, 100 and $200 \mathrm{mg} \mathrm{kg}^{-1} \mathrm{~d}^{-1}$, the serum TC level reached $11.06 \%(p<0.05), 20.74 \%(p<0.01)$, and $18.43 \%(p<0.01)$ lower than that in the HC group, respectively (Fig. 6A). EPCM-1 treatment at three dosages caused significant $(p<0.01)$ decrease in serum TC level by $19.82 \%, 20.05 \%$ and $16.36 \%$, respectively. Meanwhile, for concentrations of $50-200 \mathrm{mg} \mathrm{kg}^{-1}$ $\mathrm{d}^{-1}$, there were distinct $(p<0.01)$ reduction in TG level in mice treated with IPCM-1 by $37.19 \%, 47.93 \%$, and $42.15 \%$, respectively, and with EPCM-1 by $49.59 \%, 45.45 \%$, and $57.02 \%$, respectively, compared with HC group (Fig. 6B). Regarding LDLC level, dietary supplement of IPCM-1 at the dosage of 50, 100, and $200 \mathrm{mg} \mathrm{kg}^{-1} \mathrm{~d}^{-1}$ caused a significant $(p<0.01)$ reduction in dose-dependent manners by $26.32 \%, 38.25 \%$, and $47.37 \%$, respectively, and administration of EPCM-1 at three dosages led a significant $(p<0.01)$ reduction by $33.33 \%, 52.63 \%$, and $21.75 \%$ decrease, respectively, compared with that in HC group (Fig. 6D), thereby alleviating atherosclerotic lesions. However, no significant change in HDL-C level was observed both in IPCM-1- and EPCM-1- treated groups, indicating TC can not be transferred from peripheral tissues to the liver via the reverse 
A

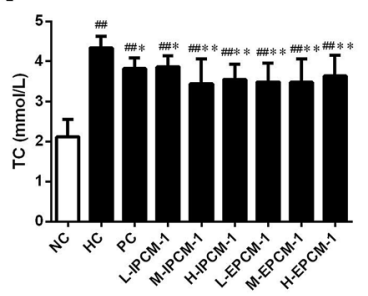

B

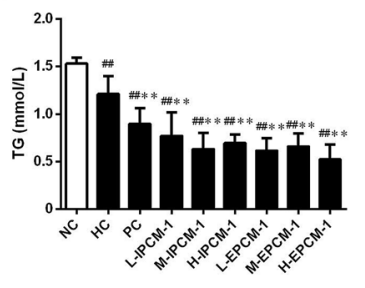

C

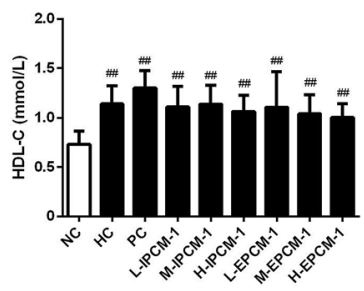

D

E
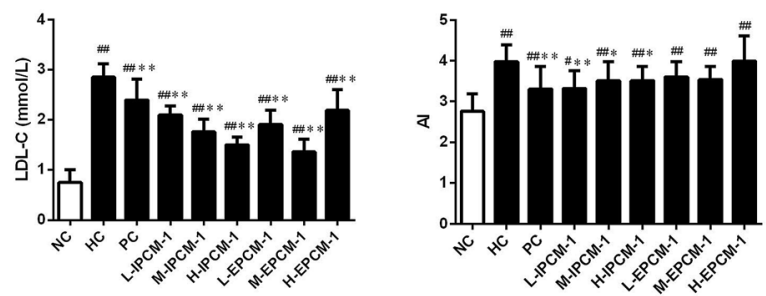

Fig. 6 Effects of IPCM-1 and EPCM-1 on serum lipid profiles in hyperlipidemic KM mice. (A) Total cholesterol (TC), (B) triglyceride (TG), (C) high density lipoprotein-cholesterol (HDL-C), (D) low density lipoprotein-cholesterol (LDL-C), (E) atherosclerosis index (Al) value. Groups: normal control $(\mathrm{NC})$, treated with standard diet; hyperlipidemic control $(\mathrm{HC})$, treated with high-fat diet; positive control $(\mathrm{PC})$, treated with high-fat diet and simvastatin $\left(10 \mathrm{mg} \mathrm{kg}^{-1} \mathrm{~d}^{-1}\right)$; L-IPCM-1, M-IPCM-1 and H-IPCM-1, treated with high-fat diet and IPCM-1 at 50, 100 and $200 \mathrm{mg} \mathrm{kg}^{-1} \mathrm{~d}^{-1}$, respectively; L-EPCM-1, M-EPCM-1 and H-EPCM-1, treated with high-fat diet and EPCM-1 at 50, 100 and $200 \mathrm{mg} \mathrm{kg}^{-1} \mathrm{~d}^{-1}$ at 50,100 and $200 \mathrm{mg} \mathrm{kg}^{-1} \mathrm{~d}^{-1}$, respectively. Data are presented as means $\pm S D, n=10 . \# p<0.05$ and $\# \# p<0.01$, values versus NC group values, $* p<0.05$ and $* * p<0.01$, values versus HC group values.

cholesterol transport pathway (Fig. 6C). More importantly, we found that at the optimal dosage, IPCM-1 (100 $\left.\mathrm{mg} \mathrm{kg}^{-1} \mathrm{~d}^{-1}\right)$ and EPCM-1 (100 mg kg ${ }^{-1} \mathrm{~d}^{-1}$ ) exerted the superior effects on reduction in serum lipid than simvastatin. The hypolipidemic activities of IPCM-1 and EPCM-1 were approximate two-fold as effective as simvastatin. Recent studies revealed that polysaccharides with relatively low molecular weight have higher biological activities. ${ }^{37-39}$ Moreover, numerous studies have demonstrated that content of glucose plays a key role in the improvement of hyperlipidemia. ${ }^{17-19}$ Owing to the large amount of IPCM-2 and EPCM-2 in IPCM-1 and EPCM-1, the bioactivity of IPCM-1 and EPCM-1 were mainly caused by IPCM-2 and EPCM-2. Combining the results of characterization of IPCM-2 and EPCM-2, we speculated that the relatively low molecular weight and the more content of glucose may explain the reason why EPCM-1 exhibited greater hypolipidemic activities than IPCM-1. Besides, AI value is an indicators of atherosclerosis and cardiovascular disease risk. ${ }^{32}$ Compared with hyperlipidemic control, IPCM-1 application showed a significant $(p<0.05)$ reduction in AI values, while dietary supplement of EPCM-1 resulted in weak reduction in AI values with no statistical significant difference (Fig. 6E).

These results are in agreement with the work of Wang and Kim, who reported that dietary supplementation of polysaccharides isolated from $C$. militaris fruiting bodies or residue could improve serum lipid profiles and serve as a protection or prevention of hyperlipidemia. ${ }^{\mathbf{1 1}, \mathbf{1 2}}$ Similarly, Dong and Yang also reported that polysaccharide-enriched fraction of $C$. militaris mycelia or culture supernatant in submerged fermentation could ameliorate the increase of serum lipid levels in hyperlipidemic rats. ${ }^{\mathbf{4 0 , 4 1}}$ Consistent with previous works, IPCM-1 and
EPCM-1 possess superior hypolipidemic properties than positive simvastatin.

\section{Effects of IPCM-1 and EPCM-1 on body weight and organ weight}

Body weight is considered as a putative indicator of health. Excess weight gain is often associated with increased fat mass, which was caused by hypertrophic and hyperplastic growth of adipocytes. ${ }^{42}$ As shown in Fig. 7A, no significant difference was detected in initial body weight of mice. However, the body weight in the HC mice was significantly $(p<0.05)$ higher than that in the NC mice after feeding high-fat diet for 14 days, but with no significant difference for 28 days presumably because of the unpleasant taste of high-fat diet. As compared with that in HC group, the mice treated with IPCM-1 (100 mg kg $\left.{ }^{-1} \mathrm{~d}^{-1}\right)$ and EPCM-1 (200 mg kg ${ }^{-1} \mathrm{~d}^{-1}$ ) for 28 days were less weight gain. These results clearly revealed that IPCM-1 and EPCM-1 were capable in control of the weight gain induced by high-fat diet, indicating that they could be applied as a future drug for weight loss. Liver is important organ for lipid metabolism. In the present study, liver indexes of HC mice were significantly higher than NC mice $(p<0.01)$ possibly because high-fat diet caused lipid accumulation in the liver. ${ }^{12,36} \mathrm{H}$-IPCM treatment trigger observable $(p<0.05)$ reduction in liver index as hepatoprotective action (Fig. 7B). Besides, IPCM-1 was also manifested distinct effects against the increase of spleen index induced by high-fat diet, which indicates that IPCM-1 exerted significant promotion with both the liver and spleen indexes (Fig. 7C). No significant difference in the organ indexes of heart and kidney was observed as a consequence of the dietary supplement of IPCM-1 and EPCM-1 as compared with HC mice (Fig. 7D and E). 
A

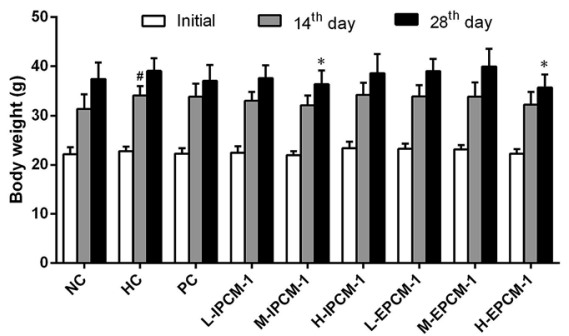

B

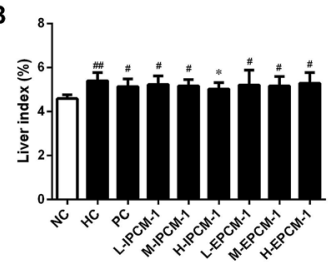

\section{$C$}

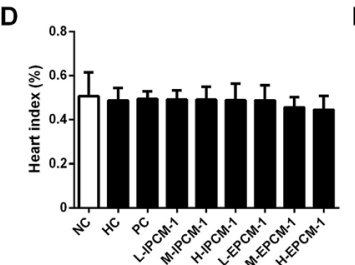

E

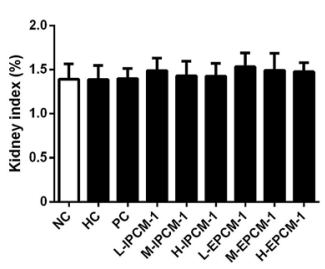

Fig. 7 Effects of IPCM-1 and EPCM-1 on body weight and organ index in hyperlipidemic KM mice. (A) Body weight, (B) liver index, (C) spleen index, (D) heart index, (E) kidney index. Groups: normal control (NC), treated with standard diet; hyperlipidemic control $(\mathrm{HC})$, treated with high-fat diet; positive control (PC), treated with high-fat diet and simvastatin (10 $\mathrm{mg} \mathrm{kg}^{-1} \mathrm{~d}^{-1}$ ); L-IPCM-1, M-IPCM-1 and H-IPCM-1, treated with high-fat diet and IPCM-1 at 50, 100 and $200 \mathrm{mg} \mathrm{kg}^{-1} \mathrm{~d}^{-1}$, respectively; L-EPCM-1, M-EPCM-1 and $\mathrm{H}$-EPCM-1, treated with highfat diet and EPCM-1 at 50,100 and $200 \mathrm{mg} \mathrm{kg}^{-1} \mathrm{~d}^{-1}$ at 50,100 and $200 \mathrm{mg} \mathrm{kg}^{-1} \mathrm{~d}^{-1}$, respectively. Data are presented as means $\pm \mathrm{SD}, n=$ 10. $\# p<0.05$ and \#\#p $<0.01$, values versus NC group values, $* p<0.05$ and $* * p<0.01$, values versus $\mathrm{HC}$ group values.

Effects of IPCM-1 and EPCM-1 on lipid regulating enzymes activities

Currently, increasing evidences have indicated that edible mushrooms are capable in lowering lipid profile, mainly due to the inhibition of the hepatic HMGR mediated cholesterol synthesis pathway, the elevation of the excretion of bile acids, and the enhancement of the removal of LDL-C from blood into liver mediated by low-density lipoprotein receptor (LDLR). ${ }^{\mathbf{4 3 - 4 5}}$ In the present study, we found hypolipidemic effects of IPCM-1 and EPCM-1 were mainly mediated by the regulation of lipid metabolic associated enzymes activities. LPL, known as a serum and liver "cleaning factor", can catalyze the decomposition of TG in CM and VLDL-C into free fatty acids, and involves in the transformation of dietary lipids into energy for peripheral tissues. ${ }^{27}$ Serum TG is an important source of fatty acids for subsequent oxidation and storage for life maintenance, and this process is closely linked to the functional capability of LPL. Kim et al. and Gao et al. reported that the hypolipidemic activities of C. militaris active constituents were attributed to their beneficial effects on the increase of serum LPL, hepatic lipase and pancreatic lipase activities. ${ }^{\mathbf{1 2 , 2 0}}$ In this study, serum LPL activities were measured, and the results are presented in Fig. 8A.

Compared with HC mice, when tested at a dose of $200 \mathrm{mg} \mathrm{kg}^{-1}$ $\mathrm{d}^{-1}$, IPCM-1 supplement remarkably increased the serum LPL activity by $55.93 \%(p<0.01)$. Serum LPL activity was dosedependently and significantly $(p<0.01)$ increased in EPCM-1treated groups and a maximum of elevation effect by $82.89 \%$ was noticed in H-EPCM group related to HC group. Meanwhile, positive simvastatin also effectively increase the serum LPL activity. As expected, we found EPCM-1 and IPCM-1 treatment significantly up-regulated the expression of serum LPL when compared with hyperlipidemic control, and this subsequently decreased serum TG levels effectively via the activation of TG hydrolysis pathway. ${ }^{20,33,46,47}$

HMGR is a glycoprotein of the endoplasmic reticulum that participates in the mevalonate pathway, and it is a rate-limiting enzyme in cholesterol synthesis pathway. ${ }^{48}$ Thus, targeting HMGR may serve as a promising therapeutic treatment for
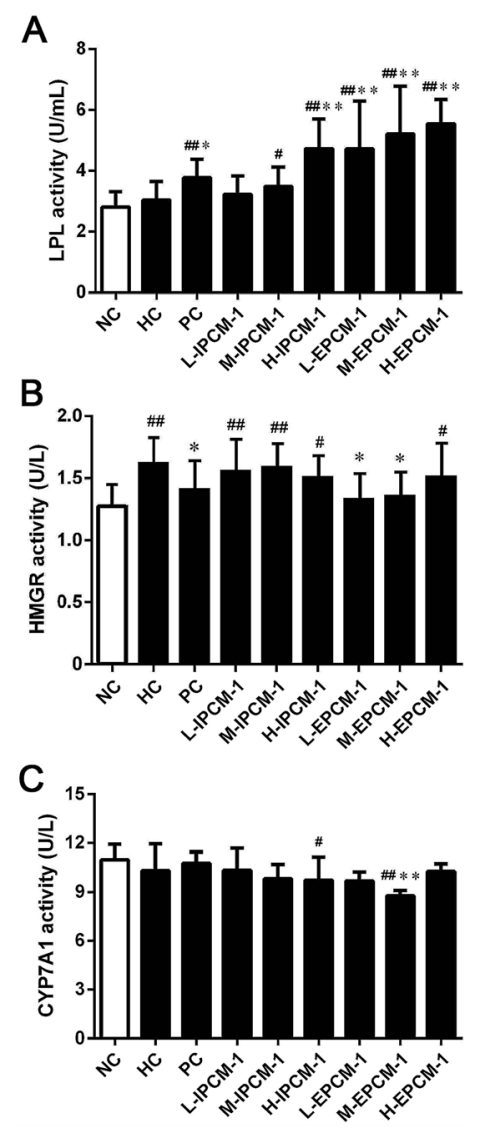

Fig. 8 Effects of IPCM-1 and EPCM-1 on serum lipoprotein lipase (LPL) activity (A), hepatic 3-hydroxy-3-methylglutaryl-CoA reductase (HMGR) activity (B) and cholesterol 7-alpha-hydroxylase (CYP7A1) activity (C) of KM mice fed a high-fat diet. Groups: normal control (NC), treated with standard diet; hyperlipidemic control $(\mathrm{HC})$, treated with high-fat diet; positive control (PC), treated with high-fat diet and simvastatin (10 mg kg $\mathrm{mg}^{-1}$ ); L-IPCM-1, M-IPCM-1 and H-IPCM-1, treated with high-fat diet and IPCM-1 at 50, 100 and $200 \mathrm{mg} \mathrm{kg}^{-1} \mathrm{~d}^{-1}$, respectively; L-EPCM-1, M-EPCM-1 and H-EPCM-1, treated with highfat diet and EPCM-1 at 50,100 and $200 \mathrm{mg} \mathrm{kg}^{-1} \mathrm{~d}^{-1}$ at 50,100 and $200 \mathrm{mg} \mathrm{kg}^{-1} \mathrm{~d}^{-1}$, respectively. Values are means $\pm \mathrm{SD}(n=10)$. \#p< 0.05 and $\# \# p<0.01$, values versus NC group values, $* p<0.05$ and $* * p$ $<0.01$, values versus $\mathrm{HC}$ group values. 


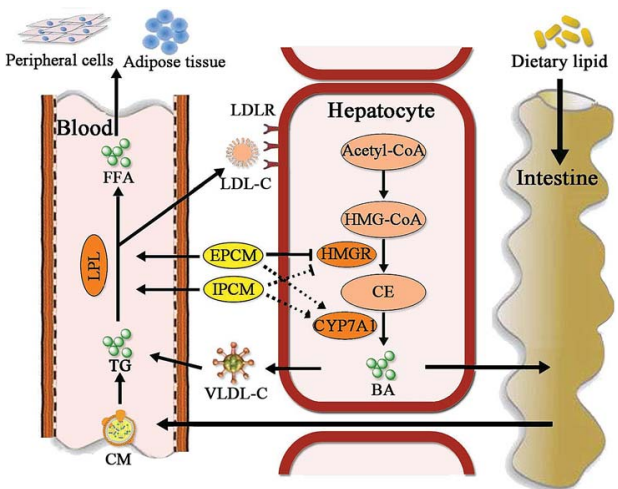

Fig. 9 Putative hypolipidemic mechanism of IPCM-1 and EPCM-1 in KM mice fed a high-fat diet. BA, bile acid; CE, cholesterol; CM, chylomicron; CYP7A1, cholesterol 7-alpha-hydroxylase; FFA, free fatty acids; HMG-CoA, 3-hydroxy-3-methyl glutaryl coenzyme A; HMGR, 3-hydroxy-3-methylglutaryl-CoA reductase; LDL-C, low-density lipoprotein-cholesterol; LDLR, low-density lipoprotein receptor; LPL, lipoprotein lipase; TG, triglycerides; VLDL-C, very low density lipoprotein-cholesterol. $\boldsymbol{f}$ represents the inhibition of polysaccharide on the enzyme; $\rightarrow$ represents the activation of polysaccharide on the

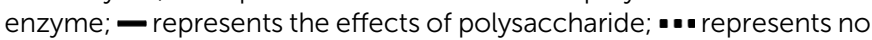
obvious effects of polysaccharide.

hyperlipidemia. In the present study, according to the results of ELISA experiments, we found the hepatic HMGR activity was remarkably lower in PC group $(p<0.05)$, compared with HC mice (Fig. 8B). Interesting, EPCM-1 treatment (50 and $100 \mathrm{mg}$ $\mathrm{kg}^{-1} \mathrm{~d}^{-1}$ ) could significantly down-regulate the expression of hepatic HMGR as effective as simvastatin, a specific inhibitors of HMGR, related to untreated mice fed with high-fat diet, which was accompanied with the optimal effect in lowering serum TC and LDL-C levels. However, dietary supplement of IPCM-1 did not influence the activity of hepatic HMGR. In this case, we thought that polysaccharides from fermented liquid, rather than mycelia of $C$. militaris, could effectively decrease plasma TC mainly through inhibiting cholesterol synthesis mediated by HMGR, ${ }^{49}$ and LDL-C transport from the serum into hepatocytes may be increased with compensatory up-regulation of LDLR induced by the low cholesterol level in hepatocytes, thereby lowering the serum LDL-C level. ${ }^{50}$

Moreover, CYP7A1 is a cytochrome P450 enzyme that catalyzes the rate-limiting reaction in bile acid synthesis pathway and converts cholesterol to 7-alpha-hydroxycholesterol. ${ }^{51}$ Thus, the elevation of hepatic CYP7A1 may be a viable strategy to lowing serum lipids levels. As shown in Fig. 8C, hepatic CYP7A1 activity in simvastatin treated group was slightly increased as compared with HC mice. At the dose we utilized, oral administration of IPCM-1 to high-fat-induced hyperlipidemic mice had no obvious effect in hepatic CYP7A1 activity. EPCM-1 application at $100 \mathrm{mg} \mathrm{kg}^{-1} \mathrm{~d}^{-1}$ caused $15.03 \%$ reduction in hepatic CYP7A1 activity with no obvious effect at 50 and $200 \mathrm{mg}$ $\mathrm{kg}^{-1} \mathrm{~d}^{-1}$ related to high-fat diet treated mice. These results revealed that the protective effects of IPCM-1 and EPCM-1 against hyperlipidemia might be attributed to the improvement of serum LPL mediated TG hydrolysis and inhibition of hepatic HMGR mediated cholesterol synthesis pathway (Fig. 9).

As mentioned above, we had successful verified the hypolipidemic activities of IPCM-1 and EPCM-1 in high-fat dietinduced hyperlipidemic mice, nevertheless, this study also has some limitations that need to be considered. Toxicity and maximum tolerated doses of IPCM-1 and EPCM-1 need followup research. Further, more in-depth investigations on the potential molecular mechanisms including the gene or protein expression related to hyperlipidemia in liver and intestine of the effects of polysaccharides from C. militaris were warranted in the future. Despite these limitations, this study was able to define the protective effects of IPCM- 1 and EPCM- 1 at different doses on complications related to hyperlipidemia in mice.

\section{Conclusions}

IPCM-1 and EPCM-1 were successfully obtained from C. militaris cultivated by submerged fermentation, and were further purified into IPCM-2 and EPCM-2, respectively. Results indicated that the average molecular weight of IPCM-2 and EPCM-2 was 32.5 and $20 \mathrm{kDa}$, respectively. IPCM-2 and EPCM-2 were mainly composed of mannose, glucose and galactose, and linked by $\alpha$-glycosidic linkage. In addition, IPCM-1 and EPCM-1, as a dietary supplement, could effectively normalize serum lipid metabolism and ameliorate symptoms of hyperlipidemia. The most effective dose of both IPCM-1 and EPCM-1 was $100 \mathrm{mg}$ $\mathrm{kg}^{-1} \mathrm{~d}^{-1}$, and EPCM-1 exerted stronger hypolipidemic properties than IPCM-1. The protective effects against hyperlipidemia might be attributed to the improvement of serum LPL mediated TG hydrolysis and inhibition of hepatic HMGR mediated cholesterol synthesis pathway. All the results suggested that IPCM and EPCM could be used as potentially natural and functional foods for the prevention and/or alleviation of hyperlipidemia.

\section{Conflicts of interest}

The authors declare that they have no competing interests.

\section{Abbreviations}

$\mathrm{AI}$

$A_{\mathrm{LPL}}$ ANOVA

BA BSA BW

$\mathrm{CD}$

$\mathrm{CM}$

C. militaris

CYP7A1

DEAE

ELISA

EPCM

FT-IR

GC-MS

$\mathrm{HC}$
Atherosclerosis index

Activity of serum LPL

Analysis of variance

Bile acids

Bovine serum albumin

Body weight

Circular dichroism

Chylomicron

Cordyceps militaris

Cholesterol 7-alpha-hydroxylase

Diethylaminoethyl

Enzyme-linked immunosorbent assay

Extracellular polysaccharides of Cordyceps militaris

Fourier transform-infrared

Gas chromatography-mass

Hyperlipidemic control 


$\begin{array}{ll}\text { HDL-C } & \text { High density lipoprotein-cholesterol } \\ \text { H-IPCM } & \text { High-dosage of IPCM } \\ \text { H-EPCM } & \text { High-dosage of EPCM } \\ \text { HMG- } & \text { 3-Hydroxy-3-methyl glutaryl coenzyme A } \\ \text { CoA } & \\ \text { HMGR } & \text { 3-Hydroxy-3-methylglutaryl-CoA reductase } \\ \text { HPGPC } & \text { High performance liquid gel permeation } \\ & \text { chromatography } \\ \text { HRP } & \text { Horseradish peroxidase } \\ \text { IPCM } & \text { Intracellular polysaccharides of Cordyceps militaris } \\ \text { LDL-C } & \text { Lipoprotein-cholesterol } \\ \text { LDLR } & \text { Low-density lipoprotein receptor } \\ \text { L-IPCM } & \text { Low-dosage of IPCM } \\ \text { L-EPCM } & \text { Low-dosage of EPCM } \\ \text { LPL } & \text { Lipoprotein lipase } \\ \text { M-IPCM } & \text { Moderate-dosage of IPCM } \\ \text { M-EPCM } & \text { Moderate-dosage of EPCM } \\ M_{\mathrm{n}} & \text { Number-average molecular weight } \\ M_{\mathrm{w}} & \text { Weight-average molecular weight } \\ \text { NAFLD } & \text { Non-alcoholic fatty liver disease } \\ \text { NC } & \text { Normal control } \\ \text { OD } & \text { Optical density } \\ \text { PBS } & \text { Phosphate buffered saline } \\ \text { PC } & \text { Positive control } \\ \text { SD } & \text { Standard deviation } \\ \text { TC } & \text { Total cholesterol } \\ \text { TG } & \text { Triglyceride } \\ \text { TMB } & 3,3^{\prime}, 5,5^{\prime} \text {-Tetramethylbenzidine } \\ \text { VLDL-C } & \text { Very low density lipoprotein-cholesterol } \\ & \end{array}$

\section{Acknowledgements}

This work was supported by the Guangdong Science and Technology Planning Project (Grant No. 2016A010105019) and the Guangzhou Science and Technology Planning Project (Grant No. 201707010138).

\section{References}

1 C. Lu, T. Sun and Y. Li, Appl. Microbiol. Biotechnol., 2018, 102, 355-366.

2 J. J. Zhang, M. Liu and Y. H. Yang, Carbohydr. Polym., 2016, 136, 588-597.

3 B. A. Golomb and E. M. A. Evans, Am. J. Cardiovasc. Drugs, 2008, 8, 373-418.

4 J. S. Lee, J. S. Kwon and D. P. Won, Carbohydr. Polym., 2010, 82, 982-988.

5 J. S. Lee, J. S. Kwon and J. S. Yun, Carbohydr. Polym., 2010, 80, 1011-1017.

6 W. Chen, G. Liu and H. Yang, Int. J. Food Eng., 2016, 13, 20160076.

7 X. J. Liu, Y. C. Huang and Y. J. Chen, RSC Adv., 2016, 6, 104094-104103.

8 L. Ma, S. Zhang and M. Du, Nutr. Res., 2015, 35, 431-439.

9 Y. S. Jing, J. H. Zhu and T. Liu, J. Agric. Food Chem., 2015, 63, 3464-3471.
10 S. K. Das, M. Masuda and A. Sakurai, Fitoterapia, 2010, 81, 961-968.

11 L. Q. Wang, N. Xu and J. J. Zhang, Carbohydr. Polym., 2015, 131, 355-362.

12 S. B. Kim, B. Ahn and M. Kim, J. Ethnopharmacol., 2014, 151, 478-484.

13 L. Ma, S. Zhang and Y. Yuan, Curr. Microbiol., 2014, 69, 852857.

14 R. M. Yu, W. Yang and L. Y. Song, Carbohydr. Polym., 2007, 70, 430-436.

15 F. Y. Wu, H. Yan and X. N. Ma, World J. Microbiol. Biotechnol., 2012, 28, 2029-2038.

16 F. Y. Wu, H. Yan and X. N. Ma, Afr. J. Microbiol. Res., 2011, 5, 2743-2751.

17 Z. Z. Ren, J. Li, N. Xu and J. J. Zhang, Carbohydr. Polym., 2017, 175, 282-292.

18 B. Yan, L. Jing and J. Wang, Carbohydr. Polym., 2015, 133, 17.

19 Z. W. Yang, J. Wang and J. G. Li, Carbohydr. Polym., 2018, 183, 11-20.

20 J. Gao, Z. Q. Lian and H. B. Zhu, Acta Pharmacol. Sin., 2011, 46, 669-676.

21 M. Huang, S. Zhang and M. L. Zhang, Appl. Microbiol. Biotechnol., 2012, 94, 763-771.

22 X. C. Liu, Z. Y. Zhu and Y. L. Tang, Carbohydr. Polym., 2016, 142, 63-72.

23 C. H. Yu, X. Y. Dai and Q. Chen, Carbohydr. Polym., 2013, 94, 56-62.

24 M. Dubois, K. A. Gilles and J. K. Hamilton, Anal. Chem., 1956, 28, 350-356.

25 M. M. Bradford, Anal. Biochem., 1976, 72, 248-254.

26 B. A. K. Rihab, B. S. Hichem and J. Neila, Carbohydr. Polym., 2017, 170, 148-159.

27 Y. Zhu, Q. Li and G. H. Mao, Carbohydr. Polym., 2014, 101, 606-613.

28 Y. Guo, S. Cong and J. Zhao, Carbohydr. Polym., 2018, 188, 54-59.

29 H. Q. Sun, Z. Y. Zhu and X. Y. Yang, RSC Adv., 2017, 7, 2387523881.

30 M. Shojaei, M. Djalali and M. Khatami, Iran. J. Kidney Dis., 2011, 5, 114-118.

31 A. Aguilera-Mendez, M. G. Hernández-Equihua and A. C. Rueda-Rocha, Nutr. Res., 2018, 57, 86-96.

32 Z. F. Wu, T. H. Gao and R. L. Zhong, Pharm. Biol., 2017, 55, 712-721.

33 S. Okamoto, T. Murano and T. Suzuki, Biochem. Biophys. Res. Commun., 2017, 486, 558-563.

34 M. Z. Sun, Y. M. Li and T. X. Wang, Carbohydr. Polym., 2018, 184, 127-134.

35 R. Ross, Nature, 1993, 362, 801-809.

36 M. E. Rinella, J. Am. Med. Assoc., 2015, 313, 2263-2273.

37 C. Z. P. Nie, P. L. Zhu and S. P. Ma, Carbohydr. Polym., 2018, 188, 236-242.

38 Y. C. Shen, W. L. Zhang and J. G. Jiang, J. Funct. Foods, 2017, 34, 118-129.

39 M. Tabarsa, S. You and E. H. Dabaghian, J. Food Drug Anal., 2017, 26, 599-608. 
40 Y. Dong, T. Jing and Q. Meng, BioMed Res. Int., 2014, 2014, 160980.

41 B. K. Yang, J. Y. Ha and S. C. Jeong, J. Microbiol. Biotechnol., 2000, 10, 784-788.

42 E. Y. Hwang, M. H. Yu and Y. S. Jung, Nutr. Res., 2016, 36, 995-1003.

43 E. Guillamón, A. Garcíalafuente and M. Lozano, Fitoterapia, 2010, 81, 715-723.

44 K. Wang, L. Bao and W. P. Xiong, J. Nat. Prod., 2015, 78, 1977-1989.

45 A. Gilramírez, V. Caz and F. R. Smiderle, J. Agric. Food Chem., 2016, 64, 1910-1920.
46 J. L. Zhang, W. Zhang and B. Mamadouba, Int. J. Biol. Macromol., 2012, 51, 504-508.

47 S. Kersten, Biochim. Biophys. Acta, 2014, 1841, 919-933.

48 Q. Q. Tao, K. Ma and Y. L. Yang, J. Org. Chem., 2016, 81, 9867-9877.

49 T. R. Pedersen and J. A. Tobert, Expert Opin. Pharmacother., 2004, 5, 2583-2596.

50 J. Z. Chen and X. F. Huang, J. Clin. Lipidol., 2009, 3, 154-158. 51 T. Q. D. Aguiar Vallim, E. J. Tarling and P. A. Edwards, Cell Metab., 2013, 17, 657-669. 